\title{
High Resolution 3-D Analysis of Quantum Dots and Other Nanostructures
}

\author{
I. Arslan ${ }^{*}$, T. J. V. Yates ${ }^{*}$, P. A. Midgley ${ }^{*}$, and N. D. Browning ${ }^{* * * * * *}$ \\ *Department of Materials Science and Metallurgy, University of Cambridge, Pembroke \\ Street, Cambridge CB2 3QZ, UK \\ ** Department of Chemical Engineering and Materials Science, University of California, One \\ Shields Ave., Davis, CA 95616, USA \\ ${ }^{* * *}$ National Center for Electron Microscopy, Lawrence Berkeley National Laboratory, One \\ Cyclotron Road, Berkeley, CA 94720, USA
}

While nanotechnology presents many exciting opportunities for materials science, it also presents significant challenges for characterization. These challenges arise as optimizing the functionality of nanoscale materials often rests with a precise control of the size, shape, crystal structure and composition of the material(s) being synthesized. While traditional techniques in transmission electron microscopy have provided feedback for the growth of single crystal films and polycrystalline solids (where crystal/defect structure/composition are the property determining factors), the application of these techniques to nanoscale particulates (where size and shape are important) is not as straightforward. However, the ability to characterize these features is now possible through electron tomography using Z-contrast imaging in the scanning transmission electron microscope (called STEM tomography) $[1,2]$.

An example of what can be achieved with these techniques is illustrated by the analysis of Sn quantum dots (QDs) embedded in a Si matrix [3]. Figure 1(a) shows a Z-contrast image of a typical quantum dot in a $\mathrm{Sn}_{0.1} \mathrm{Si}_{0.9} / \mathrm{Si}$ multilayer sample. The QDs (typically 7-10nm in size) usually consist of $\alpha$-Sn (i.e. semiconducting Sn, diamond structure, $F 4_{1} / \mathrm{d} \overline{3} 2 / \mathrm{m}$ ) and the dots' shapes resemble rhombohedrons that are bounded by the $\{111\}$ facets and where the corners are rounded by $\{110\}$ and $\{100\}$ facets. This shape, also called a tetrakaidekahedron, is known to exist as voids in silicon, and the dots outside of the layers are just these voids filled with $\mathrm{Sn} / \mathrm{Si}$ that has diffused out of the layers. Figure 1(b) shows the projection of this shape overlaid on the dot to show it is the same shape. As the crystal structure is the same for $\alpha-\mathrm{Sn}$ and $\mathrm{Si}$, this is the lowest energy structure for both systems. Two dimensional imaging can provide an estimate for what the structure is, but to truly confirm the structure and distribution of these quantum dots, a three dimensional analysis is necessary.

Figure 2(a) shows a central image of a tilt series in plan view, taken from -75 degrees to +75 degrees, and figure 2(b) shows the corresponding reconstruction in plan view. All of the dots' positions can be confirmed in the reconstruction. Figure 3(a) shows, surprisingly, that when the series was reconstructed and turned in the perpendicular direction, there were actually two layers of quantum dots! Of particular interest in determining the formation mechanism for these quantum dots is the quantum dot formed between the two layers, marked by the arrow. Figure 3(b) shows a close up reconstruction of this particular area (with the out of layer dot marked by the arrow again), and that we can still see facets on this quantum dot, that is only 3.6nm, showing the 3D spatial resolution that can be achieved. The mechanism of voidfilling/quantum dot formation will be discussed and results from other nanostructures will also be presented to highlight the limits in resolution for this method [4].

References

[1] P. A. Midgley et al, Ultramicroscopy 96, 413 (2003). 
[2] N. D. Browning et al, Nature 366, 143 (1993).

[3] Y. Lei et al, Applied Physics Letters 82, 4262 (2003).

[4] This work was supported by NSF and Royal Society fellowships and DoE DE-FG02$03 E R 46057$.

a)

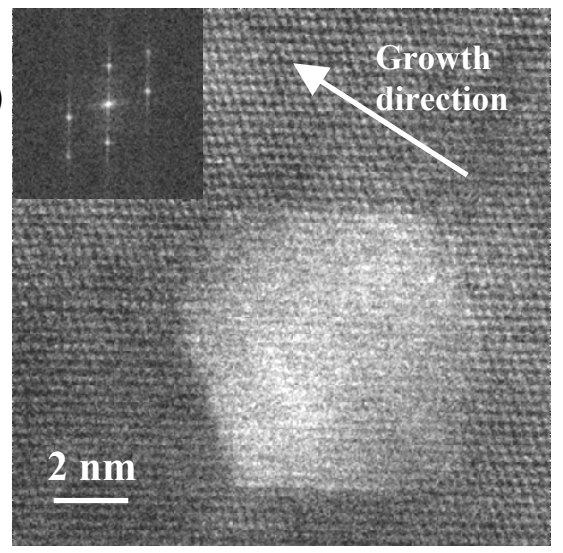

b)

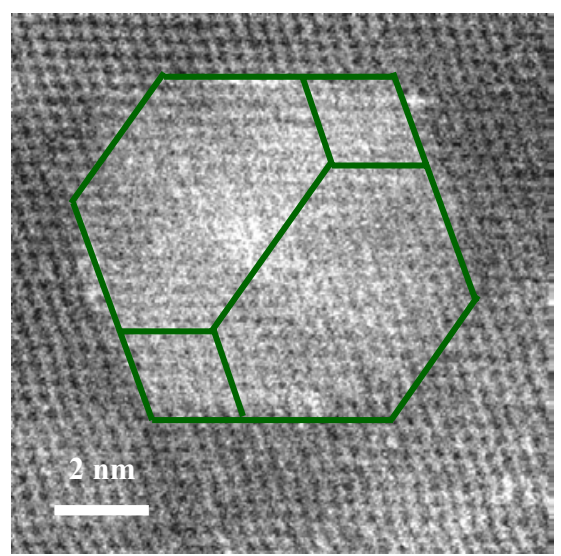

FIG. 1. Z-contrast image of (a) an $\alpha$-Sn quantum dot and (b) same dot with overlaid structure of a tetrakaidkahedron in the 110 orientation.

a)

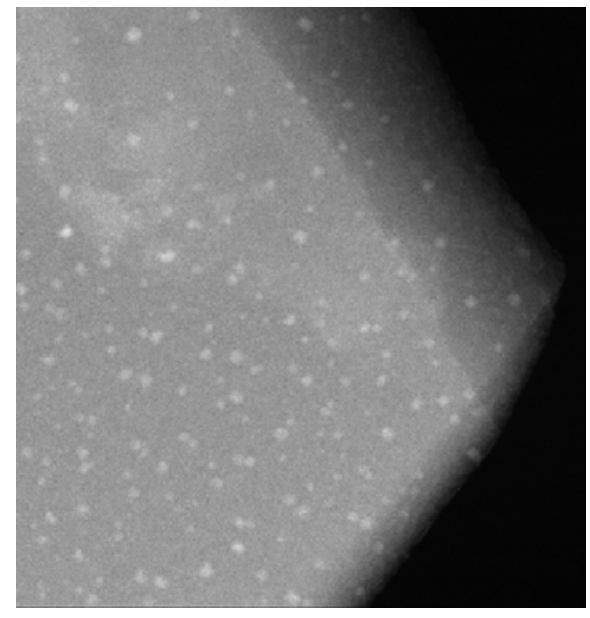

b)

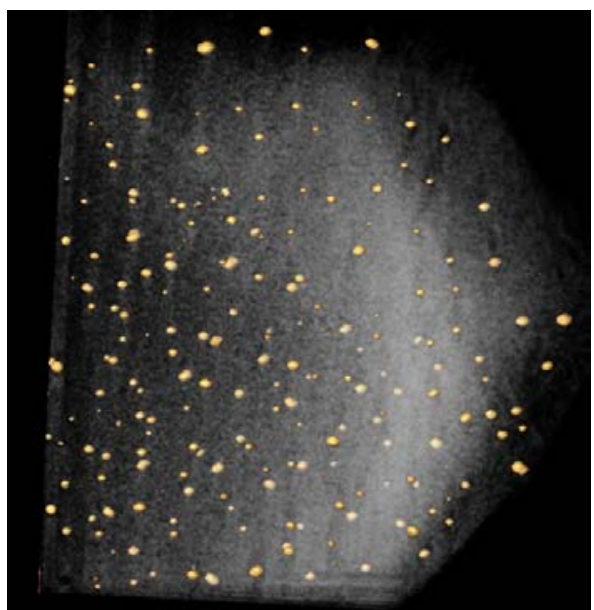

FIG. 2. (a) One Z-contrast image of a tilt series and (b) the corresponding reconstruction.

a)

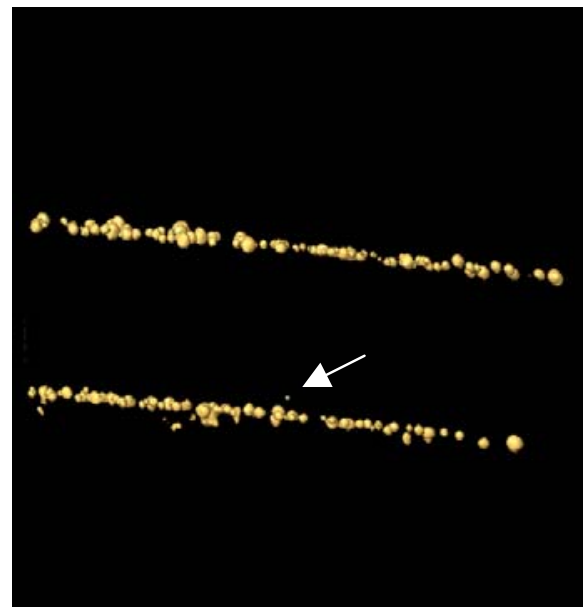

b)

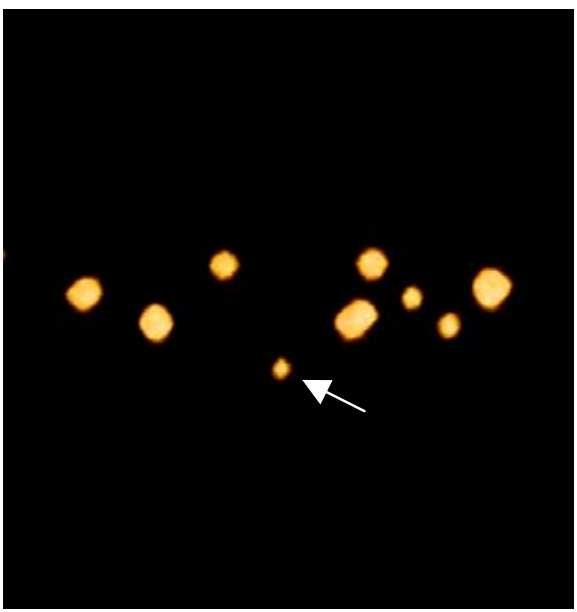

FIG. 3. (a) 110 orientation of reconstruction. (b) Close-up reconstruction of small dot showing clear faceting (arrow indicates out-of-layer dot in both figures). 